Background As part of an on-going STI microbiological surveillance programme, we investigated associations between Chlamydia trachomatis infection and the demographic, clinical, microbiological and behavioural characteristics of patients presenting with either male urethral discharge syndrome (MUDS) or vaginal discharge syndrome (VDS) to a public healthcare facility in Johannesburg, South Africa

Methods 1,218 MUDS and 1,232 VDS cases were consecutively recruited during 6 annual surveys, starting in 2007. Genital discharge pathogens were detected using a molecular assay for $N$. gonorrhoeae, Chlamydia trachomatis, Trichomonas vaginalis, Mycoplasma genitalium and by microscopy of vaginal smears (bacterial vaginosis, Candida). Serology was used to detect syphilis, HSV-2 and HIV infections. Chi-squared tests and logistic regression analyses were used to identify predictors of $C$. trachomatis infection.

Results Overall, 286 (23.5\%) men and 197 (16.0\%) women were C. trachomatis positive, with the highest prevalence observed in men $(30.5 \%)$ and women $(25.9 \%)$ aged $20-24$ years. In the multivariate analysis, C. trachomatis was less likely to be detected in MUDS patients co-infected with $N$. gonorrhoeae (aOR 0.36, 95\% CI, 0.260.49 ) and HSV-2 (aOR $0.70,95 \%$ CI, 0.51-0.95) as well as patients who had received antibiotics recently (aOR 0.43 . 95\% CI, 0.20 0.91). In contrast, the likelihood of $C$. trachomatis infection was higher in VDS patients co-infected with either $N$. gonorrhoeae (aOR 2.22 , 95\% CI, 1.48-3.32) or M. genitalium (aOR 2.24, 95\% CI, 1.45 3.47 ) infection but lower in women who were older (aOR $0.68,95 \%$ CI $0.60-0.77$ ) or who had Candida morphotypes detected (aOR $0.66,95 \%$ CI $0.44-0.98)$.

Conclusion The increased likelihood of co-existent gonococcal or $M$. genitalium infections, but the decreased likelihood of Candida infection, in women with VDS-associated chlamydial infection suggests that these women are at higher risk of STIs, and potentially

HIV. Paradoxically, gonococcal infection as well as recent antibiotic use, reduced the likelihood of MUDS patients having chlamydial infection.

\section{P3.292 THE 2013 IUSTI EUROPEAN COLLABORATIVE CLINICAL GROUP (ECCG) REPORT ON THE DIAGNOSIS AND MANAGEMENT OF CHLAMYDIAL INFECTIONS IN EUROPE}

doi:10.1136/sextrans-2013-051184.0747

B Brooks, ${ }^{1} \mathrm{~J}$ Banks, ${ }^{2} \mathrm{C}$ Ison, ${ }^{3} \mathrm{M}$ van de Laar, ${ }^{4} \mathrm{H}$ Moi, ${ }^{5} \mathrm{~K}$ Radcliffe, ${ }^{6} \mathrm{M}$ Unemo, ${ }^{5} \mathrm{~J}$ Ross, ${ }^{7} \mathrm{~J}$ White, 'R Patel. 'University of Southampton, Southampton, UK; ${ }^{2}$ Health Protection Agency, London, UK; ${ }^{3}$ European Centre for Disease Prevention and Control, Stockholm, Sweden; ${ }^{4}$ University of Oslo, Oslo, Norway; ${ }^{5}$ University Hospitals Birmingham, Birmingham, UK; ${ }^{6}$ World Health Organization Collaborating Centre for Gonorrhoea and other Sexually Transmitted Infections, Örebro University Hospital, Örebro, Sweden; ${ }^{7}$ St Thomas' Hospital London, London, UK

Background The European Collaborative Clinical Group (ECCG) is an expanding network of over 120 Sexually Transmitted Infection specialists from 36 European countries, who collaborate to conduct questionnaire based research across the European Region to identify variations in practise and inform development of international evidence-based guidelines for diagnosis and management of STIs.

The use of sensitive and specific assays and widespread screening has identified clearly the substantial burden of chlamydial infections across Europe. Infection remains common, despite established screening and treatment programmes in many European countries. Recent data has recently challenged the effectiveness of opportunistic screening and standard short course azithromycin therapy and guidance on tests of cure and partner management remain controversial.

Method The 2013 ECCG survey focuses on the diagnosis and management of chlamydial infection. An online survey constructed around clinical scenarios is gathering data on the type of test, site of testing, use of sample pooling and treatment choices. Follow up, including tests of cure and partner management is also being assessed.

Results Interim analysis of results to date show considerable variation in most of the areas studied. Despite their clear advantage many European populations are still denied the benefits of NAATs based testing. Importantly first line antibiotic choice varies as do test pooling strategies and timing and frequency of tests of cure. The survey is currently running and complete data will be available in late spring for full reporting at the conference.

Conclusion As in previous successful ECCG surveys the 2013 ECCG survey on the diagnosis and management of chlamydial infections includes a particular focus on areas where international guidance is currently lacking or poorly detailed. The ECCG has also recently expanded into parts of Eastern Europe and will be able to present data on STI care from this area for the first time.

\section{P3.293 CONDYDAV: PROSPECTIVE OBSERVATIONAL STUDY OF PATIENTS WITH EXTERNAL GENITAL WARTS(EGWS) CONSULTING IN STI CLINICS IN FRANCE}

doi:10.1136/sextrans-2013-051184.0748

IJ Chanal, ${ }^{2} \mathrm{~S}$ Fouéré, ${ }^{3} \mathrm{~F}$ Yassir Oria, ${ }^{4} \mathrm{~N}$ Spenatto, ${ }^{5} \mathrm{~F}$ Bouscarat, ${ }^{6} \mathrm{E}$ Picot, ${ }^{10} \mathrm{Aynaud}$, ${ }^{2} \mathrm{M}$ Janier, 'N Dupin, the groupe MST-Sida from the French Society of Dermatology. ${ }^{1}$ Department of Dermatology, pavillon Tarnier, Hôpital Cochin, APHP, Paris, France; ${ }^{2}$ STI centre, Hôpital Saint Louis, Paris, France; ${ }^{3}$ CIDDIST, Hôpital de la Croix Rousse, Lyon, France; ${ }^{4}$ STI centre, Hôpital de la Grave, Toulouse, France; ${ }^{5}$ CIDDIST, Hôpital Bichat, Paris, France; ${ }^{6}$ Department of Dermatology, Hôpital Saint Eloi, Montpellier, France

In this prospective, observational study, we analysed clinical features and treatments between January, 12011 and March, 312011 of patients consulting for EGWs in 15 STI clinics through France. 372 males and 111 women were included; mean age 31.2 years old. Women were younger than men $(31.7$ and 28.9 y.o respectively $\mathrm{p}<0.05) .414(85.7 \%)$ were heterosexuals, 13 bisexuals and 54 (11.2\%) homosexuals; mean age at first sexual intercourse: 17 y.o. Males reported more sex partners in the last 12 months (more than 3 partners in $32.6 \%$ versus $11.9 \% p<0.01$ ). In males, 230 had an involvement of the penis alone and 46 had an involvement of the anus alone. 76 patients had EGWs of the anus, among them 26 were MSM. In females, 76 had an infection of the vulva alone and 22 a co-infection vulva and anus. Concerning cervical cancer screening, 13 had never been tested (mean age 24.8 y.o. [17-40]); mean delay for the last screening: 12 months [1-108]. Three women have been vaccinated against HPV. 3 males were newly diagnosed HIV positives and 8 for syphilis (7 were MSM RR: 50). In females, 2 patients were HIV positives and $13 \%$ had an infection by Chlamydia trachomatis (11/84 not known: 27). Women were significantly more treated with Imiquimod (35\% Vs $19.9 \%$ p < 0.001).

\section{P3.294 ASSOCIATION OF MYCOPLASMA GENITALIUM WITH CERVICITIS IN NORTH INDIAN WOMEN ATTENDING GYNECOLOGIC CLINICS}

doi:10.1136/sextrans-2013-051184.0749

'S Sethi, ${ }^{2} \mathrm{~N}$ Rajkumari, ${ }^{3} \mathrm{~L}$ Dhaliwal, ${ }^{1}$ A Roy. 'Deptt of Microbiology, Post Graduate Institute of Medical Education and Research, Chandigarh, India; ${ }^{2}$ PGIMER, Chandigarh, India; ${ }^{3}$ Deptt of Obstetrics and Gynaecology, PGIMER, Chandigarh, India

Background The aetiology of mucopurulent cervicitis is not known in $50 \%$ of cases and cannot be attributed to the known cervical pathogens Neisseria gonorrhoeae, Chlamydia trachomatis, Genital Mycoplasmas are such organisms which can be easily overlooked as there is a lack of sufficient laboratory infrastructure for diagnosing them in developing countries. Also, there is the lack of awareness among physicians about $M$. genitalium infection. There is scarce literature showing the association of $\mathrm{M}$. genitalium with cervicitis and no reports from India. The purpose of this study was hence to 
determine the frequency of detection of $\mathrm{M}$. genitalium and to investigate causal relationship between $\mathrm{M}$. genitalium and cervicitis.

Methods Two hundred women who attended gynecologic clinics were recruited. Mucopurulent cervicitis was defined as presence of either visible yellow mucopus or presence of > 30PMNL/1000 X microscopic field on gramme stain smear of cervical mucus. First void urine (FVU), three endocervical swabs (ECS) were collected from the study group and control group. All the samples were tested by PCR amplification for presence of $\mathrm{M}$. genitalium by targeting $\mathrm{MgPa}$ gene as described previously. Samples were also subjected to culture for other genital Mycoplasmas and PCR amplification for Chlamydia trachomatis and Neisseria gonorrhoeae.

Results Mycoplasma genitalium was found in 6\% from ECS and $5 \%$ from Urine of women with Cervicitis and overall M. genitalium was detected in $11 \%$ of women and $1 \%$ from Controls $(\mathrm{P}<0.05)$. Disordered proliferative endometrium was observed in 4 of the $\mathrm{M}$. genitalium positive cases. All the patients who were positive for Chlamydia trachomatis (5\%) and Mycoplasma hominis $(7 \%)$ and Ureaplasma urealyticum(4\%) were excluded from the study. Prior miscarriage, menstrual cycle, whitish vaginal discharge and pelvic discomfort were positively associated with $\mathrm{M}$. genitalium.

Conclusions The study suggests association of $M$. genitalium infection and Cervicitis and this microorganism should be routinely screened in patients of cervicitis.

\section{P3.295 RISK BEHAVIOR AND SEXUALLY TRANSMITTED INFECTIONS AMONG PEOPLE LIVING WITH HIV/AIDS IN BELIZE, 2012}

doi:10.1136/sextrans-2013-051184.0750

'S Morales-Miranda, ${ }^{2} \mathrm{M}$ Manzanero, 'I Loya, 'R Mendizabal, 'B Alvarez. 'Universidad del Valle de Guatemala, Guatemala, Guatemala, ${ }^{2}$ Ministry of Public Health, Belize, Belize

Background STIs among people living with HIV/AIDS (PLHIV) have a direct implication on spread of HIV and the effectiveness of prevention programmes. For the first time, risk behaviour and STI prevalence was determined for PLHIV in Belize, as part of the 2012 Central American Behavioral Surveillance Survey of HIV/STI.

Methods Participants were selected based on convenience sampling of active PLWHA in the Belize Health Information System. Data collection consisted of a behavioural survey questionnaire administred through audio computer-assisted self-interview (ACASI). A blood sample was drawn for syphilis and herpes simplex virus type 2 (HSV-2) testing. Additionally, genital samples were tested for Treponema pallidum (TP), Chlamydia trachomatis (CT), Neisseria gonorrhoeae (NG), Trichomonas vaginalis (TV) and Mycoplasma genitalium (MG). Participation was voluntary and anonymous, signed consent was required. Crude proportions for categorical variables and medians and interquartile range (IOR) for numerical variables were calculated using STATA 9.0.

Results A total of 252 PLHIV were enrolled (57\% female), from three districts of Belize (concentrating 80\% of the PLHIV in the country). Median age was 45 years old (IOR 28-42). Long-standing diagnosed HIV infection was detected, median 5 years (IOR 2.3-8). Low monthly income (< US\$360) was found in $85 \%$ females and $50 \%$ males, $37.0 \%$ reported no current stable partners or occasional partners in the last year. Low percentage of consistent condom use with stable partners ( $40 \%$ female, $47.7 \%$ male) was reported. A high prevalence for HSV-2 (82.86\%), followed by TV (40.3\% female and $2.25 \%$ male) and MG (13.2\% female and $17.98 \%$ male) and a low prevalence for syphilis (1.6\%) and NG was observed (1.12\%).

Conclusions Results from the first behavioural and biological survey among PLHIV demonstrate a need for implementation of a specific Sexual Health Program for this population. This programme would include education promoting a healthy sexual lifestyle, and regular distribution of condoms.
P3.296 PREVALENCE OF CHLAMYDIAL INFECTIONS WITHIN EIGHT SOUTH AFRICAN PROVINCES (2006-2011)

doi:10.1136/sextrans-2013-051184.0751

F Radebe, V Maseko, I Basson, G de Gita, S Takuva, D A Lewis. Centre for HIV \& STIS, NICD/NHLS, Johannesburg, South Africa

Background The microbiological surveillance was undertaken in eight provinces of South Africa during 2006-2011 to determine the aetiology of the male urethritis syndrome (MUS), vaginal discharge syndrome (VDS) and genital ulcer syndrome (GUS) and the prevalence of HIV, HSV-2 and syphilis.

Methods 1361 MUS, 1691 VDS and 465 GUS cases were consecutively recruited in eight South African provinces (2006-2011). Laboratory-based diagnostic methods included nucleic acid amplification to detect Chlamydia trachomatis, Neisseria gonorrhoeae, Mycoplasma genitalium, Trichomonas vaginalis, Herpes Simplex Virus 2, Haemophilus ducreyi, Treponema pallidum and Chlamydia trachomatis serovars L1-3.

Results Overall, 202 (14.9\%) MUS and 240 (14.2\%) VDS cases were positive for $C$. trachomatis while $6(1.3 \%)$ GUS cases were positive for $C$. trachomatis serovars L1-3. The highest prevalence of $C$. trachomatis was $21.1 \%$ in Gauteng among men and $19.4 \%$ in women. The prevalence in other provinces was: Mpumalanga (men 18.4\%; women 17.4\%), Limpopo (men 14.0\%; women 16.7\%), Eastern Cape (men 16.4\%; women 13.5\%), Western Cape (men 13.5\%; women $14.9 \%$ ), Northwest (men 10.3\%; women 11.1\%), Free State (men 8.0\%; women 9.8\%) and Northern Cape (men 8.1\%; women 9.6\%). C. trachomatis serovars LI-3 prevalence was 3.2\% in the Free State, $2.8 \%$ in Mpumalanga and $0.7 \%$ in Gauteng. No C. trachomatis serovars L1-3 were detected in other five provinces.

Conclusions The prevalence of $C$. trachomatis infection was high in this population and remains an important cause of genital infection in South Africa particularly in men. This may fuel the HIV epidemic which was high in most of the provinces in this study.

\section{P3.297 SURVEILLANCE OF SYPHILIS IN THE STATE OF SAO PAULO, BRAZIL}

doi:10.1136/sextrans-2013-051184.0752

C G Luppi, W K Alencar, M A Silva, S G E Chabu, S Romera. Centro de Referência de DST/AIDS-SES-SP, São Paulo, Brazil

Background The programme of STD/AIDS in the state of São Paulo has been implementing several actions toward to control and prevent sexually transmitted infections (STI): - congenital syphilis elimination plan, - access to diagnosis and treatment of acquired syphilis; - availability of syphilis rapid test in STD clinics. The report of syphilis confirmed cases was included in the Brazilian surveillance notification system since 2010, but the surveillance of STIs in the state of São Paulo began in 1998. The objective of this study is to describe the occurrence of syphilis cases reported in the state of São Paulo.

Methods It was performed a series of all cases reported in the state of São Paulo from 1998 to June 2012.

Results From 2007 42,965 cases were reported, 59\% were male, $37 \%$ young adults, $45 \%$ had schooling up to 8 years; $49 \%$ selfreported their race/ethnicity as white. From 1998 to 20034124 cases were reported, 1447 in 2006, and 10,022 cases in 2011. The number of cases increased approximately 7 times from 2006 to 2011. It was found an increase of 132\% (586/2007-1363/2011) in the number of services which has sent the syphilis notification.

Conclusion There was a significant increase of syphilis cases reported during this period analysed in the state of Sao Paulo. This result could be explained for: - inclusion of this condition on the national list of notification, - adherence of surveillance teams to the syphilis notification, - increase of syphilis rapid test in STD services. 